\title{
EDITORIAL
}

\section{FGFR1 amplification and the progression of non-invasive to invasive breast cancer}

\author{
Alejandro A Gru* and D Craig Allred \\ See related research by Jang et al., http://breast-cancer-research.com/content/14/4/R115
}

\begin{abstract}
The incidence of invasive breast cancer (IBC) can be dramatically reduced by improving our abilities to detect and treat ductal carcinoma in situ (DCIS). Progress will be based on a detailed understanding of molecular mechanisms responsible for tumor progression. An interesting study by Jang and colleagues evaluated and compared the frequency of amplification of four oncogenes (HER2, C-MYC, CCND1 and FGFR1) in large cohorts of pure DCIS, in the DCIS component of IBC, and in corresponding IBC. Of particular interest, they found a twofold increase in FGFR1 amplification in IBC versus pure $D C I S$, and significantly reduced disease-free survival in amplified versus unamplified IBC - leading the authors to conclude that FGFR1 plays an important role in the development and progression of IBC. These observations indeed provide hints that FGFR1 is important in this setting, although the issue is very complex and far from resolved.
\end{abstract}

Invasive breast cancer (IBC) evolves through a series of increasingly abnormal premalignant stages, over decades in most cases [1,2]. Ductal carcinoma in situ (DCIS) is a late stage in this evolution and the immediate precursor for most IBC. Currently, about 60,000 new cases of DCIS are diagnosed in the USA each year [3]. If undetected, at least one-third of cases will progress to IBC [4]. About 200,000 cases of IBC are also diagnosed [3], and nearly all evolve from DCIS that was not detected.

The incidence of IBC can be dramatically reduced by improving our abilities to detect and successfully treat DCIS, which will be based on a detailed understanding of molecular mechanisms responsible for tumor progression.

*Correspondence: agru@path.wustl.edu

Department of Pathology \& Immunology, Washington University in St. Louis School of Medicine, St. Louis, MO 63110, USA
Although there is much to learn, recent studies have begun to shed light on this important issue [5,6]. Among these is an interesting study by Jang and colleagues described in a recent issue of Breast Cancer Research, which evaluated and compared the frequency of amplification of four oncogenes (HER2, c-MYC, CCND1, and FGFR1) in large cohorts of pure DCIS $(n=175)$, in the DCIS component of IBC $(n=203)$, and in the corresponding IBC $(n=427)$ [1]. Amplification was carefully assessed by fluorescence in situ hybridization on tissue microarrays containing triplicate $2 \mathrm{~mm}$ cores/sample, which is far more tissue than used in most tissue microarray studies. Overall, they found reasonable rates of amplification for each oncogene in IBC consistent with many previous studies [7]. Far fewer studies of DCIS are available for comparison.

The main focus of the study was to compare amplification between pure DCIS and IBC, hypothesizing that differences may help identify genes that are important in the transition from in situ to invasive disease. In this regard, the most notable findings included significantly higher rates of HER2 amplification in pure DCIS versus IBC ( $31 \%$ vs. $20 \% ; P=0.004)$, which has been shown before [8], and significantly lower rates of FGFR1 in pure DCIS versus IBC (6\% vs. $13 \%$; $P=0.02$ ), which is novel. These differences were more pronounced in lesions of high histological grade (HER2 60\% vs. 34\%; FGFR1 7\% vs. 16\%). Amplification frequencies in intrinsic molecular subtypes of IBC were also generally consistent with previous studies [7]. Jang and colleagues also looked at the relationship between amplification and clinical outcome. In these studies, amplification of FGFR1 was associated with significantly reduced disease-free survival in patients with IBC (about $10 \%$ at 8 years), particularly in hormone-receptor-positive patients, although $H E R 2$, $c-M Y C$, and $C C N D 1$ were not prognostic in this cohort.

The twofold elevation of FGFR1 amplification in IBC versus pure DCIS, and the poor prognosis in IBC, led the authors to conclude that activation plays an important role in the progression of breast cancer, including, in particular, the in situ to invasive transition. This is a reasonable conclusion in the sense that it is also 
consistent with previous studies showing that FGFR1 activation is oncogenic for breast cancer in transgenic mice [9], and is associated with increased invasion of breast cancer cell lines in vitro [10] and poor prognosis in IBC $[7,11,12]$, especially in receptor-positive disease $[11,12]$.

Although our understanding of FGFR 1 at the molecular level is incomplete, important aspects are known about its function and the consequences of gene amplification [13]. For example, the gene encodes a tyrosine kinase receptor that is part of a large family of fibroblast growth factors and receptors. In a normal setting, activation of FGFR1 can lead to transactivation of mitogen-activated protein kinase and $\mathrm{AKT}$, which collectively are essential for breast development, including the growth and differentiation of luminal epithelial cells. However, increased FGFR1 activity, such as occurs through gene amplification, can result in increased luminal cell proliferation and, in transgenic mouse models, this hyperplasia may eventually evolve into in situ and invasive mammary carcinomas.

In humans, the chromosomal region where FGFR1 resides, $8 \mathrm{p} 11.2$, is amplified in significant proportions of many types of cancers, including kidney cancer, lung cancer, prostate cancer, and leukemias. The region is also amplified in 10 to $20 \%$ of IBC [13]. However, the 8 p11.2 amplicon is large and complex, and FGFR1 is only one of several candidate oncogenes within the region [14] others include LSM1, PPAPDC1B, WHSC1L1, and BAG4, which are also important in breast development and cell cycle regulation, among other relevant functions. To complicate matters more, only about $50 \%$ of FGFR1amplified tumors appear to overexpress the transcript [15].

The study by Jang and colleagues provides additional evidence of an important role for FGFR1 amplification in the progression of IBC [1]. The study also provides tantalizing hints that FGFR1 may be important in the in situ to invasive transition, which is a critical step in the progression of a nonlethal to potentially lethal disease and this aspect of the study is entirely novel. Having said this, we need to be cautious about drawing conclusions regarding gene function and malfunction based on correlative studies of this nature alone. For example, the main evidence that FGFR1 is important in this study is elevated amplification in IBC versus DCIS. By analogous reasoning, the elevated amplification of HER2 observed in DCIS versus IBC could be taken as evidence for suppression of tumor progression, which we know is wrong. Determining the molecular function, oncogenic potential, and clinical significance of FGFR1 at any stage of breast cancer evolution will require many additional comprehensive laboratory and clinical studies. These studies are particularly worthwhile in the sense that we have effective drugs to inhibit FGFR1 activity [13]. Jain and Turner have summarized recent interesting data in terms of the functional biology of the fibroblast growth factor receptors and development of inhibitors of these molecules, with emphasis on challenges to successfully target this pathway in breast cancer [16]. These could lead to new strategies for preventing the progression of DCIS to IBC, or restoring responsiveness of receptorpositive IBC to endocrine therapy if activation is confirmed to induce resistance $[11,12]$ - perhaps FGFR1 will be the next HER2.

\section{Abbreviations}

DCIS, ductal carcinoma in situ; FGFR1, fibroblast growth factor receptor 1; HER2, human epidermal growth factor receptor 2 ; IBC, invasive breast cancer.

\section{Competing interests}

The authors declare that they have no competing interests.

Published: 14 November 2012

\section{References}

1. Jang MH, Kim EJ, Choi Y, Lee HE, Kim YJ, Kim JH, Kang E, Kim SW, Kim IA, Park SY: FGFR1 is amplified during the progression of in situ to invasive breast carcinoma. Breast Cancer Res 2012, 14:R115.

2. Allred DC: Biological features of human premalignant breast disease and the progression to cancer. In Diseases of the Breast. Edited by Harris JR, Lippman ME, Morrow M, Hellman S, Osborne CK. 4th edition. New York: Lippincott Williams and Wilkins; 2009:323-334

3. American Cancer Society: Breast Cancer Facts and Figures; Atlanta, GA: American Cancer Society Inc.; 2011-2012.

4. Allegra CJ, Aberle DR, Ganschow P, Hahn SM, Lee CN, Millon-Underwood S, Pike MC, Reed SD, Saftlas AF, Scarvalone SA, Schwartz AM, Slomski C, Yothers G, Zon R: National Institutes of Health State-of-the-Science Conference statement: Diagnosis and Management of Ductal Carcinoma In Situ September 22-24, 2009. J Natl Cancer Inst 2010, 102:161-169.

5. Polyak K: Breast cancer: origins and evolution. J Clin Invest 2007, 117:3155-3163.

6. Lee S, Stewart S, Nagtegaal I, Luo J, Wu Y, Colditz G, Medina D, Allred DC: Differentially expressed genes regulating the progression of ductal carcinoma in situ to invasive breast cancer. Cancer Res 2012, 72:4574-4586.

7. Letessier A, Sircoulomb F, Ginestier C, Cervera N, Monville F, Gelsi-Boyer V, Esterni B, Geneix J, Finetti P, Zemmour C, Viens P, Charafe-Jauffret E, Jacquemier J, Birnbaum D, Chaffanet M: Frequency, prognostic impact, and subtype association of $8 p 12,8 q 24,11 q 13,12 p 13,17 q 12$, and $20 q 13$ amplifications in breast cancers. BMC Cancer 2006, 6:245.

8. Latta EK, Tjan S, Parkes RK, O'Malley FP: The role of HER2/neu overexpression/amplification in the progression of ductal carcinoma in situ to invasive carcinoma of the breast. Mod Pathol 2002, 15:1318-1325.

9. Welm BE, Freeman KW, Chen M, Contreras A, Spencer DM, Rosen JM: Inducible dimerization of FGFR1: development of a mouse model to analyze progressive transformation of the mammary gland. J Cell Bio/ 2002, 157:703-714

10. Xian W, Schwertfeger KL, Vargo-Gogola T, Rosen JM: Pleiotropic effects of FGFR1 on cell proliferation, survival, and migration in a 3D mammary epithelial cell model. $J$ Cell Bio/ 2005, 171:663-673.

11. Elbauomy Elsheikh S, Green AR, Lambros MB, Turner NC, Grainge MJ, Powe D, Ellis IO, Reis-Filho JS: FGFR1 amplification in breast carcinomas: a chromogenic in situ hybridisation analysis. Breast Cancer Res 2007, 9:R23.

12. Turner N, Pearson A, Sharpe R, Lambros M, Geyer F, Lopez-Garcia MA, Natrajan R, Marchio C, lorns E, Mackay A, Gillett C, Grigoriadis A, Tutt A, Reis-Filho JS, Ashworth A: FGFR1 amplification drives endocrine therapy resistance and is a therapeutic target in breast cancer. Cancer Res 2010, 70:2085-2094.

13. Schwertfeger KL: Fibroblast growth factors in development and cancer: insights from the mammary and prostate glands. Curr Drug Targets 2009, 10:632-644.

14. Gelsi-Boyer V, Orsetti B, Cervera N, Finetti P, Sircoulomb F, Rouge C, Lasorsa L, Letessier A, Ginestier C, Monville F, Esteyriès S, Adélaïde J, Esterni B, Henry C, 
Ethier SP, Bibeau F, Mozziconacci MJ, Charafe-Jauffret E, Jacquemier J,Bertucci F, Birnbaum D, Theillet C, Chaffanet M: Comprehensive profiling of 8p11-12 amplification in breast cancer. Mol Cancer Res 2005, 3:655-667.

15. Gru AA, Salavaggione AL, Hoog J, Snyder J, Tao Y, Ellis M, Allred DC: Amplification of FGFR1 gene and its relationship with gene expression and clinical significance in breast cancer. Modern Pathol 2010, 48a:206.

16. Jain VK, Turner NC: Challenges and opportunities in the targeting of fibroblast growth factor receptors in breast cancer. Breast Cancer Res 2012, $14: 208$

\section{doi:10.1186/bcr3340}

Cite this article as: Gru AA, Allred DC: FGFR1 amplification and the

progression of non-invasive to invasive breast cancer. Breast Cancer Research 2012, 14:116. 